\title{
On Consensus Clustering Validation
}

\author{
João M.M. Duarte ${ }^{1,2}$, Ana L.N. Fred ${ }^{1}$, André Lourenço $^{1}$, and F. Jorge F. Duarte ${ }^{2}$ \\ 1 Instituto de Telecomunicações, Instituto Superior Técnico, Lisboa, Portugal \\ \{jduarte, afred, arlourenco\}@lx.it.pt \\ 2 GECAD - Knowledge Engineering and Decision Support Group, \\ Instituto Superior de Engenharia do Porto, Porto, Portugal \\ $\{$ jod, fjd\}@isep. ipp.pt
}

\begin{abstract}
Work on clustering combination has shown that clustering combination methods typically outperform single runs of clustering algorithms. While there is much work reported in the literature on validating data partitions produced by the traditional clustering algorithms, little has been done in order to validate data partitions produced by clustering combination methods. We propose to assess the quality of a consensus partition using a pattern pairwise similarity induced from the set of data partitions that constitutes the clustering ensemble. A new validity index based on the likelihood of the data set given a data partition, and three modified versions of well-known clustering validity indices are proposed. The validity measures on the original, clustering ensemble, and similarity spaces are analysed and compared based on experimental results on several synthetic and real data sets.
\end{abstract}

\section{Introduction}

Clustering ensemble approaches have been proposed aiming to improve data clustering robustness and quality [1], reuse clustering solutions [2], and cluster data in a distributed way. Schematically, these methods can be split into two main phases: the construction of the clustering ensemble (CE); and the combination of information extracted from the $\mathrm{CE}$ into a consensus partition. The Evidence Accumulation Clustering method (EAC) [1] additionally produces, as an intermediate result, a learned pairwise similarity between patterns, summarized in a co-association matrix. In the literature on this topic, one can find many alternative ways of building the clustering ensemble, defining the combination strategy and extraction algorithm, and choosing the final number of clusters. All these lead to a myriad of alternative clustering solutions. Hence, we are faced with the following problem: "for a given data set, which clustering solution should be selected?".

While there is much work reported in the literature on validating data partitions produced by the traditional clustering algorithms [3], little has been done in order to validate data partitions produced by clustering combination methods. Most of the reported works use measures of consistency between consensus solutions and the clustering ensemble, such as Average Normalized Mutual Information [2] and Average Cluster Consistency [4]. The classical validity indices may also be used to assess the quality of the consensus partition. This requires the original data representation to be available,

E.R. Hancock et al. (Eds.): SSPR \& SPR 2010, LNCS 6218, pp. 385-394, 2010.

(C) Springer-Verlag Berlin Heidelberg 2010 
which may not always be possible. Also, not considering clustering ensemble information should be a drawback, since the clustering structure, used by the clustering combination methods to produce the consensus partitions, is not used.

In this paper we propose the validation of clustering combination results at three levels:

- original data representation - measure the consistency of clustering solutions with the structure of the data, perceived from the original representation (either featurebased or similarity-based);

- clustering ensemble level - measure the consistency of consensus partitions with the clustering ensemble;

- learned pairwise similarity - measure the coherence between clustering solutions and the co-association matrix induced by the clustering ensemble.

Additionally to the methodology of evaluation at these distinct levels, we propose a new criterion based on likelihood estimates, and adaptation of "classical" cluster validity measures to pairwise similarity representations.

The remaining of the paper is organized as follows. Section 2 formulates the clustering ensemble problem, and describes the EAC method, that will be used in our experiments. The methodology for the validation of consensus partitions is presented in section 3. In section 4, a new validity index based on pairwise similarities is proposed. Experiments comparing all the validation measures are presented in section 5. Finally, the conclusions appear in section 6

\section{Clustering Combination}

Let $\mathcal{X}=\left\{x_{1}, \cdots, x_{n}\right\}$ be a data set with $n$ data patterns. Different partitions of $\mathcal{X}$ can be obtained by using different clustering algorithms, changing parameters and/or initializations for the same clustering algorithm, using different subsets of data features or patterns, projecting $\mathcal{X}$ to subspaces, and combinations of these. A clustering ensemble, $\mathcal{P}$, is defined as a set of $N$ data partitions of $\mathcal{X}$ :

$$
\mathcal{P}=\left\{P^{1}, \cdots, P^{N}\right\}, P^{l}=\left\{C_{1}^{l}, \cdots, C_{K^{l}}^{l}\right\},
$$

where $C_{k}^{l}$ is the $k^{\text {th }}$ cluster in data partition $P^{l}$, which contains $K^{l}$ clusters. Different partitions capture different views of the structure of the data. Clustering ensemble methods use a consensus function $f$ which maps a clustering ensemble $\mathcal{P}$ into a consensus partition $P^{*}=f(\mathcal{P})$.

The Evidence Accumulation Clustering method (EAC) [1] considers each data partition $P^{l} \in \mathcal{P}$ as an independent evidence of data organization. The underlying assumption of EAC is that two patterns belonging to the same "natural" cluster will be frequently grouped together. A vote is given to a pair of patterns every time they cooccur in the same cluster. Pairwise votes are stored in a $n \times n$ co-association matrix, $\mathbf{C}$, normalized by the total number of combined data partitions, i.e., $\mathbf{C}_{i j}=\frac{\sum_{l=1}^{N} v_{0} e_{i j}^{l}}{N}$ where vote $_{i j}^{l}=1$ if $x_{i}$ and $x_{j}$ co-occur in a cluster of data partition $P^{l}$; otherwise vote $_{i j}^{l}=0$. The consensus partition is obtained by applying some clustering algorithm over the co-association matrix, $\mathbf{C}$. 


\section{Consensus Partition Validation}

We herein propose the assessment of the quality of a consensus partition, $P^{*}$, by measuring its consistency at three levels: the original representation space; the clustering ensemble; and the learned pairwise similarity.

\subsection{Validity Measures on the Original Data Space}

Validity measures on the original data space are the most common approaches to perform clustering validation. The basic idea consists of evaluating a data partition using a utility or cost function, and comparing it with other partitions of the same data set. The utitlity/cost function usually measures the intra-cluster compactness and inter-cluster separation of a given data partition. Many different validity measures on the original data representation space have been proposed in the literature [3]. In this paper we will focus on three of them: the Silhouette, Dunn's and Davies-Bouldin indices.

Let $\mathcal{X}=\left\{x_{1}, \cdots, x_{n}\right\}$ be the data set, $P=\left\{C_{1}, \cdots, C_{K}\right\}$ its partition into $K$ clusters, and $\left|C_{l}\right|$ the number of elements in the $l$-th cluster. Let $d\left(x_{i}, x_{j}\right)$ be the dissimilarity (distance) between data patterns $x_{i}$ and $x_{j}$.

The Silhouette index [5] is formally defined as follows. Let $a_{i}$ denote the average distance between $x_{i} \in C_{l}$ and the other patterns in the same cluster, and $b_{i}$ the minimum average distance between $x_{i}$ and all patterns grouped in another cluster:

$$
a_{i}=\frac{1}{\left|C_{l}\right|-1} \sum_{\substack{x_{j} \in C_{l} \\ j \neq i}} d\left(x_{i}, x_{j}\right), \quad b_{i}=\min _{k \neq l} \frac{1}{\left|C_{k}\right|} \sum_{x_{j} \in C_{k}} d\left(x_{i}, x_{j}\right) .
$$

The silhouette width, $s_{i}$, for each $x_{i}$, produces a score in the range $[-1,1]$ indicating how well $x_{i}$ fits in its own cluster when compared to other clusters; the global Silhouette index, $S$, is given by the average silhouette width computed over all samples in the data set:

$$
s_{i}=\frac{b_{i}-a_{i}}{\max \left\{a_{i}, b_{i}\right\}}, \quad S=\frac{1}{n} \sum_{i=1}^{n} s_{i}
$$

Dunn's index, quantifying how well a set of clusters represent compact and separated clusters [6], is defined as:

$$
D=\frac{\min _{1 \leq q \leq K} \min _{1 \leq r \leq K, r \neq q} \operatorname{dist}\left(C_{q}, C_{r}\right)}{\max _{1 \leq p \leq K} \operatorname{diam}\left(C_{p}\right)}
$$

where $\operatorname{dist}\left(C_{q}, C_{r}\right)$ represents the distance between clusters $C_{q}$ and $C_{r}$, and $\operatorname{diam}\left(C_{p}\right)$ is the $p^{\text {th }}$ cluster diameter:

$$
\operatorname{dist}\left(C_{q}, C_{r}\right)=\min _{x_{i} \in C_{q}, x_{j} \in C_{r}} d\left(x_{i}, x_{j}\right), \quad \operatorname{diam}\left(C_{p}\right)=\max _{x_{i}, x_{j} \in C_{p}} d\left(x_{i}, x_{j}\right) .
$$

The best partition is the one that maximizes the index value, $D$. 
Davies-Bouldin index [7], is defined as the ratio of the sum of within-cluster scatter and the value of between-cluster separation:

$$
D B=\frac{1}{K} \sum_{k=1}^{K} \max _{m \neq k}\left\{\frac{\Delta\left(C_{k}\right)+\Delta\left(C_{m}\right)}{d\left(\nu_{k}, \nu_{m}\right)}\right\}, \Delta\left(C_{k}\right)=\frac{\sum_{x_{i} \in C_{k}} d\left(x_{i}, \nu_{k}\right)}{\left|C_{k}\right|}
$$

where $\Delta\left(C_{k}\right)$ is the average distance between all patterns in $C_{k}$ and their cluster center $\nu_{k}=\frac{\sum_{x_{i} \in C_{k}} x_{i}}{\left|C_{k}\right|}$. Small values of $D B$ correspond to clusters that are compact, and whose centers are far away from each other. The data partition that minimizes $D B$ is the optimal one.

\subsection{Validity Measures on the Clustering Ensemble Space}

These validity indices rely on the agreement between the consensus partition, $P^{*}$, and the partitions in the clustering ensemble $\mathcal{P}=\left\{P_{1}, \cdots, P_{N}\right\}$.

Let $H(P)=-\sum_{k=1}^{K} p(k) \log p(k)$ be the entropy of data partition $P$, with $p(k)=$ $\frac{n_{k}}{n}$, and $n_{k}$ the number of patterns in the $k^{\text {th }}$ cluster of $P$. The mutual information between two data partitions, $P^{*}$ and $P^{l}$, is defined as:

$$
M I\left(P^{*}, P^{l}\right)=\sum_{i}^{K^{*}} \sum_{j}^{K^{l}} \frac{p(i, j)}{p(i) p(j)},
$$

with $p(i, j)=\frac{1}{n}\left|C_{i}^{*} \cap C_{j}^{l}\right|$, the fraction of shared samples in clusters $C_{i}^{*}$ and $C_{j}^{l}$. Strehl and Ghosh [2] define the Average Normalized Mutual Information as:

$$
\operatorname{ANMI}\left(P^{*}, \mathcal{P}\right)=\frac{1}{N} \sum_{l=1}^{N} \frac{M I\left(P^{*}, P^{l}\right)}{\sqrt{\left.H\left(P^{*}\right) H\left(P^{l}\right)\right)}} .
$$

Higher values of $A N M I\left(P^{*}, \mathcal{P}\right)$ suggest better quality consensus partitions.

The Average Cluster Consistency [4] (ACC) is another validity measure based on the similarity between the partitions of the clustering ensemble and the consensus partition. The main idea consists of measuring how well the clusters $C_{m}^{l}$ of the clustering ensemble fit in a cluster $C_{k}^{*}$ of the consensus partition. If all patterns $x_{i} \in C_{m}^{l}$ belong to the same cluster $C_{k}^{*}$, for all clusters of the clustering ensemble, then the average cluster consistency between the consensus partition and the clustering ensemble is perfect. The ACC measures the similarity between two partitions, $P^{*}$ and $P^{l}$, based on a weighting of shared samples in matching clusters:

$$
\operatorname{sim}\left(P^{*}, P^{l}\right)=\frac{1}{n} \sum_{m=1}^{K^{l}} \max _{1 \leq k \leq K^{*}}\left|C_{k}^{*} \cap C_{m}^{l}\right|\left(1-\frac{\left|C_{k}^{*}\right|}{n}\right),
$$

where $K^{l} \geq K^{*}$. Note that cluster intersection, $\left|C_{k}^{*} \cap C_{m}^{l}\right|$, is weighted by $\left(1-\frac{\left|C_{k}^{*}\right|}{n}\right)$ in order to prevent high similarity values in situations where $P^{*}$ has a few clusters with almost all the data patterns. The drawback is that consensus partitions with balanced 
cluster cardinality are preferred. The ACC is defined as the average similarity between each data partition in the clustering ensemble $\left(P^{l} \in \mathcal{P}\right)$ and the consensus partition $P^{*}$ :

$$
A C C\left(P^{*}, \mathcal{P}\right)=\frac{1}{N} \sum_{i=1}^{N} \operatorname{sim}\left(P^{i}, P^{*}\right) .
$$

From a set of possible choices, the best consensus partition is the one that achieves the highest $A C C\left(P^{*}, \mathcal{P}\right)$ value.

\subsection{Validity Measures on a Similarity Space}

In the following, modifications of the validity indices presented in subsection 3.1 are proposed, aiming to accommodate the same principles to a pairwise similarity representation. Consider a pairwise similarity measure $s\left(x_{i}, x_{j}\right)$ between pairs of patterns $\left(x_{i}, x_{j}\right)$. In this paper, we will define $s\left(x_{i}, x_{j}\right)=\mathbf{C}_{i j}$, the pairwise similarity induced from the clustering ensemble [1], summarized in matrix $\mathbf{C}$ (see section 2).

In order to compute a Silhouette-like validity index in a similarity space, we propose to measure the within-cluster compactness and the inter-cluster separability adapting the formulas defined in equation 2 as below:

$$
a_{s_{i}}=\frac{1}{\left|C_{l}\right|-1} \sum_{\substack{x_{j} \in C_{l} \\ j \neq i}} s\left(x_{i}, x_{j}\right), \quad b_{s_{i}}=\max _{k \neq l} \frac{1}{\left|C_{k}\right|} \sum_{x_{j} \in C_{k}} s\left(x_{i}, x_{j}\right) .
$$

While in equation 2 low values for $a_{i}$ and high values for $b_{i}$ corresponded to high cluster compactness and separation, in equation 11 it is the opposite since we are using similarities. In this case, high values for $a_{s_{i}}$ and low values for $b_{s_{i}}$ imply good data partitions. For this reason, the numerator of equation 3 (left) is changed for the computation of the silhouette width, being defined as:

$$
s_{s_{i}}=\frac{a_{s_{i}}-b_{s_{i}}}{\max \left\{a_{s_{i}}, b_{s_{i}}\right\}} .
$$

The average silhouette width using similarities is then computed as $S_{s}=\frac{1}{n} \sum_{i=1}^{n} s_{s_{i}}$.

For Dunn's index, the similarity between the $q^{\text {th }}$ and the $r^{\text {th }}$ clusters, and the diameter of $C_{p}$ were redefined:

$$
\operatorname{sim}\left(C_{q}, C_{r}\right)=\max _{x_{i} \in C_{q}, x_{j} \in C_{r}} s\left(x_{i}, x_{j}\right), \quad \operatorname{diam}_{\mathrm{S}}\left(C_{p}\right)=\min _{x_{i}, x_{j} \in C_{p}} s\left(x_{i}, x_{j}\right) .
$$

By the fact that we are using similarities instead of distances, we take the inverse of equation 4 to define a Dunn-like validation index:

$$
D_{s}=\frac{\min _{1 \leq p \leq K} \operatorname{diam}_{\mathrm{s}}\left(C_{p}\right)}{\max _{1 \leq q \leq K} \max _{1 \leq r \leq K, r \neq q} \operatorname{sim}\left(C_{q}, C_{r}\right)+1} .
$$

Since the information regarding the cluster centers $\left\{\nu_{1}, \cdots, \nu_{K}\right\}$ is not available in a similarity-based data representation, in our adaptation of the Davies and Bouldin's 
validity index, it was necessary to introduce a new concept of center of a cluster. In order to incorporate pairwise similarities instead of the original vectorial data representation, we estimate the central pattern $\nu_{k}$ of cluster $C_{k}$ as the element with maximum mean similarity within each cluster (innermost pattern), as defined below.

$$
\nu_{k}=\underset{x_{i} \in C_{k}}{\arg \max } \sum_{\substack{x_{j} \in C_{k} \\ j \neq i}} s\left(x_{i}, x_{j}\right)
$$

Davies and Bouldin's validity index is redefined as

$$
D B_{s}=\frac{1}{K} \sum_{k=1}^{K} \max _{m \neq k}\left\{\frac{s\left(\nu_{k}, \nu_{m}\right)}{\Delta_{s}\left(C_{k}\right)+\Delta_{s}\left(C_{m}\right)}\right\}
$$

where $\Delta_{s}\left(C_{k}\right)$ is the average similarity between all patterns in $C_{k}$.

\section{Statistical Validity Index Based on Pairwise Similarity}

We now propose a new validity index to assess the quality of $P^{*}$ based on the likelihood of the data constrained to the data partition, $L\left(\mathcal{X} \mid \mathrm{P}^{*}\right)$, assessed from pairwise similarities, as per in the co-association matrix, $\mathbf{C}$, defined in section 2 .

Our work is inspired in the Parzen-window density estimation technique [8] with variable size window, also known as K-nearest neighbor density estimation. This technique estimates the probability density of pattern $x, p(x)$, within a region $R$ with volume $V_{R}$. The volume $R$ is defined as a function of the $K_{N}$ nearest neighbors of $x$, i.e., $V_{R}$ is the volume enclosed by the region that contains all the $K_{N}$ nearest neighbors of $x$. The probability density $p(x)$ is estimated as $\hat{p}(x)=\frac{K_{N}}{n V_{R}}$.

The new validity measure based on the likelihood of the data $\mathcal{X}$ (assuming $x \in \mathcal{X}$ to be independent and identically-distributed random variables) given a partition $P$, is defined as:

$$
L(\mathcal{X} \mid P)=\prod_{i=1}^{N} p\left(x_{i} \mid P\right), \quad p\left(x_{i} \mid P\right)=\sum_{k=1}^{K} p\left(x_{i} \mid C_{k} \in P\right) \cdot \operatorname{Pr}\left(C_{k}\right) .
$$

Following the idea behind the Parzen-window density estimation method, we define the probability density of $x_{i}$ given cluster $C_{k}$ as:

$$
p\left(x_{i} \mid C_{k}\right)=\frac{K_{N}}{\left|C_{k}\right| \cdot V_{k}\left(x_{i}\right)}
$$

where $V_{k}\left(x_{i}\right)$ represents the volume of a sufficiently small region that contains all the patterns of the neighborhood $K N N_{k}\left(x_{i}\right) \bigcup\left\{x_{i}\right\}$, and $K N N_{k}\left(x_{i}\right)$ is the set of the $K_{N}$ most similar data patterns to $x_{i}$ in cluster $C_{k}$. Since we rely only on pairwise similarities, as induced from the clustering ensemble, we approximate the intrinsic volume $V_{k}\left(x_{i}\right)$ by a quantity proportional to it, defined by:

$$
V_{k}\left(x_{i}\right) \triangleq \operatorname{diam}_{k}\left(x_{i}\right), \quad \operatorname{diam}_{k}\left(x_{i}\right)=2\left(1-\min _{x_{j} \in K N N_{k}\left(x_{i}\right)} \mathbf{C}_{i j}\right)
$$


where $\operatorname{diam}_{k}\left(x_{i}\right)$ represents the "diameter" of the region centered at $x_{i}$ that contains the neighborhood of $x_{i}$. Since the similarity matrix, $\mathbf{C}$, takes values in the interval $[0 ; 1]$, the above transformation $1-\mathbf{C}_{i j}$ leads to a dissimilarity measure; the diameter thus corresponds to twice the dissimilarity of the $K_{N}^{\text {th }}$ nearest neighbor of $x_{i}$.

Using equations 17, 18 and estimating $\operatorname{Pr}\left(C_{k}\right)$ as $\frac{1}{n}\left|C_{k}\right|$, the likelihood of the data set $\mathcal{X}$ given a data partition $P$ is defined as:

$$
L(\mathcal{X} \mid P)=\prod_{i=1}^{N} \sum_{k=1}^{K} \frac{K_{N}}{n \cdot V_{k}\left(x_{i}\right)} .
$$

The underlying reasoning for using $L$ as a validity index is the following.

Given a clustering ensemble, the co-association matrix, $\mathbf{C}$, corresponds to the maximum likelihood estimate of the probability of pairwise co-occurrence of patterns in a cluster. Taking this co-occurrence probability as the pattern pairwise similarity induced by the $\mathrm{CE}$, the likelihood of the data set $\mathcal{X}$ given a combination partition $P^{*}$ is estimated by $L\left(\mathcal{X} \mid P^{*}\right)$. The statistical validity index based on the pairwise similarity, $L$, thus corresponds to a goodness of fit of the combined partition, $P^{*}$, with the clustering ensemble and the pairwise information extracted from it. Best combination strategies should therefore lead to highest likelihood values, $L$, of the data.

In a similar way, we can compute the likelihood of the data given the combination partition using the original data representation space. In this case, the likelihood $L$ corresponds to a goodness of fit of the combined partition, $P^{*}$, with the statistical properties of the data on the original representation. In the following we denote by $L_{O}$ the likelihood computed from the original data representation, and by $L_{S}$ the likelihood computed from the co-association matrix (induced similarity).

\section{Experimental Results}

Five real (available at the UCI repository http://archive.ics.uci.edu/ml) and nine synthetic data sets were used to assess the performance of the validity measures on a wide variety of situations, including data sets with arbitrary cluster shapes, different cardinality and dimensionality, well-separated and touching clusters, and distinct cluster densities. The Iris data set consists of 50 patterns from each of three species of iris flowers, characterized by four features. The Std Yeast is composed of 384 patterns (normalized to have 0 mean 0 and unit variance) characterized by 17 features, split into 5 clusters concerning 5 phases of the cell cycle. The Optdigits is a subset of Handwritten Digits data set containing only the first 100 patterns of each digit, from a total of 3823 data samples characterized by 64 attributes. The House Votes data set is composed of two clusters of votes for each of the U.S. House of Representatives Congressmen on the 16 key votes identified by the Congressional Quarterly Almanac. From a total of 435 (267 democrats and 168 republicans) only the patterns without missing values were considered, resulting in 232 patterns (125 democrats and 107 republicans). The Wine data set consists of the results of a chemical analysis of wines grown in the same region in Italy divided into three clusters with 59, 71 and 48 patterns described by 13 features. Both House Votes and Wine data sets were normalized to have unit variance. The synthetic data sets are shown in figure 1 


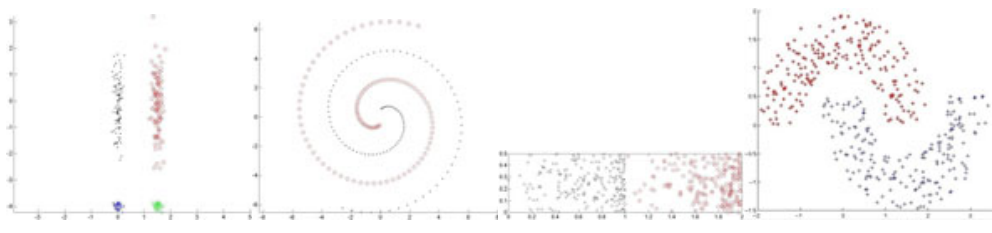
(a) Cigar
(b) Spiral
(c) Bars
(d) 2 Half Rings

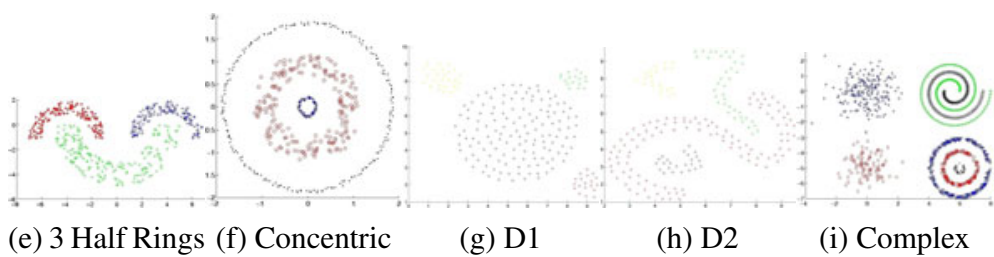

Fig. 1. Synthetic data sets

Table 1. $N M I\left(P^{*}, P^{0}\right)$ for the consensus partitions selected by each validity measure

\begin{tabular}{|c|c|c|c|c|c|c|c|c|c|c|c|c|c|c|c|c|c|c|c|c|c|c|}
\hline & \multicolumn{11}{|c|}{ Clustering Ensemble Construction Method A } & \multicolumn{11}{|c|}{ Clustering Ensemble Construction Method B } \\
\hline Data Set & $L_{O}$ & $S_{O}$ & $D_{o}$ & $D B_{O}$ & $L_{S}$ & $S_{s}$ & $D_{s}$ & $D B_{S}$ & ANMI & ACC & Best & $L_{O}$ & $S_{O}$ & $D_{o}$ & $D B_{O}$ & $L_{s}$ & $S_{S}$ & $D_{s}$ & $D B_{S}$ & ANMI & ACC & Best \\
\hline Iris & 0.81 & 0.81 & 0.71 & 0.71 & 0.81 & 0.81 & 0.71 & 0.81 & 0.81 & 0.81 & 0.81 & 0.81 & 0.81 & 0.72 & 0.72 & 0.81 & 0.81 & 0.72 & 0.72 & 0.81 & 0.81 & 0.81 \\
\hline Std $Y$ & & 0.49 & 0.08 & 0.53 & .49 & 0.53 & 0.24 & 0.0 & 0.49 & 0.49 & $l_{0}$ & 48 & 0.48 & 0.37 & 0.32 & 1.48 & 0.53 & 0.23 & 0.48 & .48 & 0.48 & 0.53 \\
\hline Optd & & 0.81 & 0.71 & 0 & 81 & 0.81 & 0.63 & 0.81 & 81 & 0.81 & 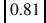 & 81 & 0.83 & 0.83 & 0.72 & 81 & 0.81 & 0.72 & 0 & .81 & .83 & 83 \\
\hline House Votes & 0.50 & 0.50 & 0.03 & 0.03 & 0.50 & 0.14 & 0.14 & 0.1 & .50 & 0.50 & 0. & .49 & 0.49 & 0.02 & 0 & .49 & 0.49 & 0.14 & 0.14 & 0.49 & 0.49 & 49 \\
\hline Win & 0.77 & 0.77 & 0.66 & 0.08 & 0.77 & 0.66 & 0.08 & 0.66 & 0.77 & 0.77 & 0.77 & .77 & 0.80 & 0.06 & 0.17 & 0.80 & 0.77 & 0.17 & 0.06 & 0.77 & 0.77 & 0.80 \\
\hline Cigar & & 1.00 & 1.00 & 0.23 & 1.00 & 1.00 & 1.00 & 1.00 & 1.00 & 1.00 & 1.00 & .84 & 1.00 & 1.00 & 1.00 & 84 & 1.00 & 0.38 & 1.00 & .84 & 0.84 & 1.00 \\
\hline Spiral & & 0.00 & 0.05 & 0.05 & 1.00 & 0.00 & 1.00 & 1.00 & 0.00 & 0.00 & 1.00 & 01 & 0.01 & 0.08 & & 01 & 0.01 & 1.00 & & 01 & 0.01 & 1.00 \\
\hline Bars & & 0.94 & 0.06 & 0.06 & 0.94 & 0.94 & 0.06 & 0.94 & 0.94 & 0.94 & 0. & 0.94 & 0.94 & 0.21 & 0 . & .94 & 0.94 & 0.21 & 0.21 & 0.94 & 0.94 & 0.94 \\
\hline 2 Half Rings & 0.99 & 0.99 & 0.17 & 0.17 & 0.99 & 0.99 & 0.99 & 0.9 & 0.99 & 0.99 & 0.9 & .87 & 0.99 & 0.21 & 0. & 0.87 & 0.87 & 0.99 & 0. & 0.87 & 0.87 & 0.99 \\
\hline 3 Half $\mathrm{R}$ & 1.00 & 1.00 & 0.08 & 0.08 & 1.00 & 1.00 & 1.00 & 1.00 & 1.00 & 1.00 & 1.00 & .00 & 1.00 & 1.00 & 1.00 & 1.00 & 1.00 & 1.00 & 1.00 & 1.00 & 1.00 & 1.00 \\
\hline Concentric & 1.00 & 1.00 & 0.09 & 0.09 & 1.00 & 1.00 & 1.00 & 1.00 & 1.00 & 1.00 & 1.00 & 0.70 & 1.00 & 0.14 & 0.14 & 0.70 & 0.70 & 1.00 & 1.00 & 0.70 & 0.70 & 1.00 \\
\hline $\mathbf{I}$ & 1.00 & 1.00 & 1.00 & 0.05 & 1.00 & 1.00 & 1.00 & 1.00 & 1.00 & 1.00 & 1.00 & 0.40 & 1.00 & 1.00 & 1. & 0.40 & 1.00 & 1.00 & 1. & 1.00 & 1.00 & 1.00 \\
\hline & 1 & 1.00 & 0.14 & 0.12 & 1.00 & 1.00 & 1.0 & 1. & 1.00 & 1.00 & 1.00 & 0.57 & 0.71 & 0. & 0.34 & 57 & 0.57 & 1. & 1. & 0.71 & 0.71 & 1.00 \\
\hline Com & 0.83 & 0.44 & 0.83 & 0.44 & 0.83 & 0.83 & 0.82 & 0.82 & 0.83 & 0.83 & 0. & 0.70 & 0.70 & 0.70 & 0.63 & 0.70 & 0.63 & 0.56 & 0.70 & 0.70 & 0.70 & 0.87 \\
\hline 3est criterion & 13 & 11 & 3 & 1 & 13 & 11 & 7 & 10 & 12 & 12 & & 5 & 11 & 5 & 4 & 6 & 7 & 6 & & 6 & 7 & \\
\hline
\end{tabular}

For each data set, two different methods were used to build the clustering ensembles. In the first method (A), the $K$-means algorithm was used to produce $N=150$ data partitions, each one with exactly $K=20$ clusters for the Iris data set, $K=50$ for the Concentric data sets, $K=120$ for the Complex data set, and $K=30$ for all the other data sets. In the second method (B), the $K$-means algorithm was also used to build clustering ensembles with the same size, but the number of clusters for each data partition was randomly chosen to be an integer in the interval $[10 ; 30]$. The clustering ensemble construction method A (leading to $\mathcal{P}^{A}$ ) is expected to be a "good" clustering ensemble, in the sense that its clusters have less probability of mixing patterns from different "natural" clusters than the clustering ensemble construction method $\mathrm{B}\left(\mathcal{P}^{B}\right)$, since $K^{l}, \forall P_{l} \in \mathcal{P}^{A}$ is always higher than $\min _{P^{l} \in \mathcal{P}^{B}} K^{l}$, with the exception of the Iris data set. The consensus partitions were obtained applying the EAC method using the Single-Link, Average-Link, Complete-Link, Centroid-Link and Ward-Link hierarchical clustering algorithms at the final step. $K_{N}$ was defined as $\lceil\sqrt{n}\rceil$.

Table 1 shows the $N M I\left(P^{*}, P^{0}\right)$ values between the best data partition $P^{*}$, according to each validity measure, and the "real" (ground-truth) data partition $P^{0}$. The 


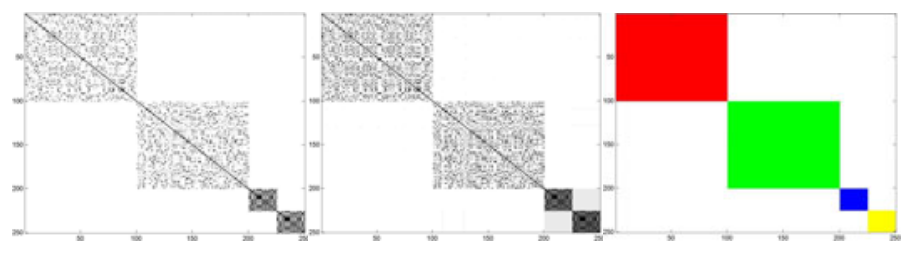

(a)

(b)

(c)

Fig. 2. Co-association matrices for (a) CE construction method A, (b) CE construction method B and (c) "natural" partition of data, for Cigar data set

subscripts ${ }_{o}$ and $s$ point out that the validity measure was evaluated on the original space or the similarity space, respectively. The columns designated by "Best" indicate the value of NMI for the best obtained consensus partition. In order to use the criterion based on the likelihood estimates $(L)$ on the original space, the diameter of a region was computed as $\operatorname{diam}\left(x_{i}\right)=2 \max _{x_{j} \in K N N\left(x_{i}\right)} d\left(x_{i}, x_{j}\right)$, using the Euclidean distance to measure dissimilarity, and $K N N\left(x_{i}\right)$ corresponds to the set of the $K_{N}$ closest patterns to $x_{i}$. The results for the clustering ensemble construction method A show that the $L$ validity measure had the best performance, both on the original and similarity spaces, selecting the best consensus partition in 13 out of 14 data sets, followed by $A N M I$ and $A C C$ criteria with 12 , and $S_{O}$ and $S_{S}$ with 11 . While $L_{o}$ and $L_{s}$ selected the same partitions, $S_{o}$ and $S_{s}$ had different choices on several data sets. The performances of $D$ and $D B$ were better on the pairwise similarity space than on the original space, suggesting that the first should be preferred. For the clustering ensemble construction method B, $S$ on the original space was the best validity measure, being the best criterion in 11 data sets. $D B$ was the best on the similarity space by selecting in 9 data sets equal or better partitions than the other indices. $L$ was the best criterion only 5 times on the original space and 6 on the similarity space. The poor performance of $L$ is due to its sensibility to "bad" clustering ensembles. Figure 2 shows the co-association matrices for construction methods A and B and the "natural" partition for the Cigar data set. While in figure 2 (a) there are no co-associations between patterns belonging to different "natural" clusters, in figure 2 (b) it can be seen (especially on the lower right corner) that some patterns from distinct "natural" clusters have co-association different from 0 . This explains why the $L_{s}$ performed correctly on the clustering ensemble construction method A and not on B.

From the comparison involving the criteria on the original and similarity spaces, we conclude that $L$ (on both spaces) is the best choice if the clustering ensemble is "good", $S$ is robust on the original space, and $D$ was the worst criterion (despite that the similarity space version presents better results than the original space version). We also conclude that the consensus partition evaluation may also be restricted to the co-association matrix. This has the advantages of exploring sparse similarities representations (particularly when using $L_{s}$ ) and complying with data privacy. Evaluating consensus partitions on the original space has also another disadvantage: how to validate a consensus partition if the partitions belonging to the clustering ensemble were produced using different representations (e.g. distinct subset of feature, random projections, etc)? 
By comparing the criteria on the similarity spaces with the criteria based on the consistency between the clustering ensemble partitions and the consensus partition, $L_{s}$ was better than ANMI and ACC in construction method $\mathrm{A}$, and $D B_{s}$ was better in construction method B; so we can discard both ANMI and ACC, and rely instead on the similarity-based criteria in order to assess the consensus partitions.

\section{Conclusions}

The validation of clustering solutions were proposed at three distinct levels: original data representation, learned pairwise similarity, and consistency with the clustering ensemble partitions. A new validity measure based on the likelihood estimation of pattern pairwise co-occurrence probabilities was introduced. Experimental results seem to indicate that: the new validity measure is a good choice for performing consensus clustering validation when the clusters belonging to the clustering ensemble are not likely to contain patterns of different "natural" clusters; the learned similarity-based criteria can be used, instead of the traditional clustering ensemble measures; and the similarity-based criteria are a good option when the original data representation is not available. More extensive evaluation of the validity indices is being conducted over a larger number of data sets and on the comparison of consensus results produced by different combination strategies.

\section{Acknowledgments}

We acknowledge financial support from the FET programme within the EU FP7, under the SIMBAD project (contract 213250). This work was partially supported by the Portuguese Foundation for Science and Technology (FCT), Portuguese Ministry of Science and Technology, under grant PTDC/EIACCO/103230/2008.

\section{References}

1. Fred, A., Jain, A.: Combining multiple clustering using evidence accumulation. IEEE Trans. Pattern Analysis and Machine Intelligence 27(6), 835-850 (2005)

2. Strehl, A., Ghosh, J.: Cluster ensembles - a knowledge reuse framework for combining multiple partitions. J. Mach. Learn. Res. 3, 583-617 (2003)

3. Halkidi, M., Batistakis, Y., Vazirgiannis, M.: On clustering validation techniques. Intelligent Information Systems Journal 17(2-3), 107-145 (2001)

4. Duarte, F.J., Duarte, J.M.M., Rodrigues, M.F.C., Fred, A.L.N.: Cluster ensemble selection using average cluster consistency. In: KDIR 2009: Proc. of Int. Conf. on Knowledge Discovery and Information Retrieval (October 2009)

5. Rousseeuw, P.: Silhouettes: a graphical aid to the interpretation and validation of cluster analysis. Journal of Computational and Applied Mathematics 20, 53-65 (1987)

6. Dunn, J.C.: A fuzzy relative of the isodata process and its use in detecting compact, well separated clusters. Cybernetics and Systems 3(3), 32-57 (1974)

7. Davies, D., Bouldin, D.: A cluster separation measure. IEEE Transaction on Pattern Analysis and Machine Intelligence 1(2) (1979)

8. Duda, R.O., Hart, P.E., Stork, D.G.: Pattern Classification, 2nd edn. Wiley-Interscience, Hoboken (November 2000) 